J. Korean Math. Soc. 49 (2012), No. 1, pp. 49-67

http://dx.doi.org/10.4134/JKMS.2012.49.1.049

\title{
$\ell$-RANKS OF CLASS GROUPS OF FUNCTION FIELDS
}

\author{
Sunghan Bae And Hwanyup Jung
}

\begin{abstract}
In this paper we give asymptotic formulas for the number of $\ell$-cyclic extensions of the rational function field $\mathrm{k}=\mathbb{F}_{q}(T)$ with prescribed $\ell$-class numbers inside some cyclotomic function fields, and density results for $\ell$-cyclic extensions of $\mathrm{k}$ with certain properties on the ideal class groups.
\end{abstract}

\section{Introduction}

Let $\mathbb{Q}$ be the field of rational numbers and $\ell$ be a prime number. In the 1980 s F. Gerth studied extensively the asymptotic behavior of $\ell$-cyclic extensions of $\mathbb{Q}$ with certain conditions on the ideal class groups and ramified primes. Let us recall Gerth's results more precisely. Write $N_{s, x}$ for the number of $\ell$-cyclic extensions of $\mathbb{Q}$ with conductor $\leq x$ and $\ell$-class number $\ell^{s}$. In [5], it is shown that to obtain an asymptotic formula for $N_{s, x}$, it suffices to count the number $M_{s+1, x}$ of $\ell$-cyclic extensions of $\mathbb{Q}$ whose conductor is $\leq x$ and divisible by exactly $s+1$ distinct primes, and whose $\ell$-class number is $\ell^{s}$. In [6], a matrix $M$ over $\mathbb{F}_{\ell}$ is associated to each $\ell$-cyclic extension $F$ of $\mathbb{Q}$ with $s+1$ ramified primes such that the $\ell$-class number of $F$ is $\ell^{s}$ precisely when $\operatorname{rank}(M)=s$, and an asymptotic formula for $N_{s, x}$ is given by studying the asymptotic behavior of the number of such matrices. In [8], for $\ell=2$, an effective algorithm for computing the density $d_{t, e}$ (resp. $d_{t, e}^{\prime}$ ) of the quadratic fields with 4-class rank $e$ (in the narrow sense) in the set of imaginary (resp. real) quadratic fields with $t$ ramified primes, and explicit formulas for their limiting densities $d_{\infty, e}=\lim _{t \rightarrow \infty} d_{t, e}$ and $d_{\infty, e}^{\prime}=\lim _{t \rightarrow \infty} d_{t, e}^{\prime}$ are given. An explicit formula for the limiting density $d_{\infty, e}$, which depends only on $\ell$ and $e$, is given in [10] for an arbitrary prime number $\ell$. Similar results for $\ell^{n}$-cyclic extensions of $\mathbb{Q}$ with prescribed (narrow) genus groups are given in [9].

Let $\mathrm{k}=\mathbb{F}_{q}(T)$ be the rational function field over the finite field $\mathbb{F}_{q}$. Let $\ell$ be a prime number different from the characteristic of $\mathrm{k}$ and $r$ be the smallest

Received August 10, 2010; Revised July 11, 2011.

2010 Mathematics Subject Classification. 11R58, 11R29, 11R45.

Key words and phrases. class groups, function fields.

The first author was supported by SRC program (ASARC R11-2007-035-01001-0).

The second author was supported by the Korea Science and Engineering Foundation(KOSEF) grant funded by the Korea government(MEST) (No. 2009-0071789). 
positive integer such that $\ell \mid q^{r}-1$. In this article we study analogous problems for $\ell$-cyclic extensions of $\mathrm{k}$ inside some cyclotomic function fields. The content of this paper is as follows. In $\S 1$ we recall several asymptotic formulas in $\mathbb{A}=\mathbb{F}_{q}[T]$, which can be found in [11] and [12]. In $\S 2$ we recall the genus theory for function fields [2] and extend some results of Wittmann [13] to the narrow case. In $\S 3.1$ we give an asymptotic formula for the number $N_{s, r n}$ of $\ell$-cyclic extensions $F$ inside some cyclotomic function fields with $\ell$-class number $\ell^{s}$ and with conductor $N$ of degree $r n$ in the case $r>1$. Similar results in the case $r=1$ are given in $\S 3.2$. In $\S 4$ we give the density for $\ell$-ranks in $\ell$-cyclic function fields. In $\S 5$ we give a generalization of $\S 4$ to $\ell^{m}$-cyclic extensions of $\mathrm{k}$ inside some cyclotomic function fields.

\section{Some asymptotic formulas in $\mathbb{A}=\mathbb{F}_{q}[T]$}

In this section we recall several asymptotic formulas in $\mathbb{A}=\mathbb{F}_{q}[T]$, which will be used later in this paper. For the details and proofs we refer to [11] and $[12]$.

- $P(n):=$ the set of monic irreducible polynomials in $\mathbb{A}$ of degree $n$, and $p(n)=|P(n)|$. Then

$$
p(n)=\frac{q^{n}}{n}+O\left(\frac{q^{n / 2}}{n}\right) \quad([11, \text { Chap. 8], [12, Theorem 2.2]). }
$$

- $P(n, k):=$ the set of all square-free monic polynomials of degree $n$ with $k$-irreducible factors, and $p(n, k)=|P(n, k)|$. Then

$$
p(n, k)=\frac{q^{n}(\log n)^{k-1}}{(k-1) ! n}+O\left(\frac{q^{n}(\log n)^{k-2}}{n}\right) \quad([11, \text { Theorem 9.9]). }
$$

- $P_{r}(r n, k):=$ the set of all square-free monic polynomials of degree $r n$ with $k$-irreducible factors whose degrees are divisible by $r$, and $p_{r}(r n, k)$ $=\left|P_{r}(r n, k)\right|$. Following the method of $[11, \S 9]$,

$$
p_{r}(r n, k)=\frac{q^{r n}(\log n)^{k-1}}{(k-1) ! r^{k} n}+O\left(\frac{q^{r n}(\log n)^{k-2}}{n}\right) .
$$

Intuitively, (1.3) follows from (1.2) and that the probability that a prime whose degree is divisible by $r$ is $\frac{1}{r}$. For $A, M \in \mathbb{A}$, relatively prime,

- $P(n, A, M):=$ the set of monic irreducible polynomials of degree $n$ which are congruent to $A$ modulo $M$, and $p(n, A, M)=|P(n, A, M)|$. Then

$$
p(n, A, M)=\frac{q^{n}}{\phi(M) n}+O\left(\frac{q^{n / 2}}{n}\right) \quad([12, \text { Theorem 4.8]), }
$$

where $\phi(M)=\left|(\mathbb{A} / M \mathbb{A})^{\times}\right|$. 
Also, for a nontrivial Dirichlet character $\chi$, we have

$$
\sum_{P, \operatorname{deg} P=n} \chi(P)=O\left(\frac{q^{n / 2}}{n}\right) \quad([12, \S 4(4),(5)]) .
$$

From (1.1), we have

$$
\begin{gathered}
\sum_{P, \operatorname{deg} P \leq n} \frac{\operatorname{deg} P}{q^{\operatorname{deg} P}}=n+O(1), \\
\sum_{P, r \mid \operatorname{deg} P \leq n r} \frac{\operatorname{deg} P}{q^{\operatorname{deg} P}}=n+O(1), \\
\sum_{P, \operatorname{deg} P \leq n} \frac{1}{q^{\operatorname{deg} P}}=\log n+O(1), \\
\sum_{P, r \mid \operatorname{deg} P \leq n r} \frac{1}{q^{\operatorname{deg} P}}=\frac{\log n}{r}+O(1) .
\end{gathered}
$$

From $(1.2),(1.3)$ and the partial summation formula, we have

$$
\begin{gathered}
\sum_{d=1}^{n} \sum_{P \in P(d, k)} \frac{1}{q^{d}} \sim \frac{(\log n)^{k}}{k !}, \\
\sum_{d=1}^{n} \sum_{P \in P_{r}(r d, k)} \frac{1}{q^{r d}} \sim \frac{(\log n)^{k}}{k ! r^{k}} .
\end{gathered}
$$

One more asymptotic formula which will be used later is

$$
\sum_{m_{1}+\cdots+m_{k}=n} \frac{1}{m_{1} \cdots m_{k}} \sim \frac{k(\log n)^{k-1}}{n} .
$$

\section{Genus theory for function fields}

Write $\infty$ for the place of $\mathrm{k}$ associated to $1 / T$. Let $\mathrm{k}_{\infty}$ be the completion of $\mathrm{k}$ at $\infty$, i.e., $\mathrm{k}_{\infty}=\mathrm{k}((1 / T))$. Let $\Omega=\mathrm{k}_{\infty}(\sqrt[q-1]{-1 / T})$. We only consider those function fields which can be embedded into $\Omega$. For a monic polynomial $M$ of $\mathbb{A}, \mathrm{k}_{M}$ denotes the cyclotomic function field of conductor $M$ (see $[12, \S 12]$ ). Any abelian extension $F$ of $\mathrm{k}$ inside $\Omega$ is contained in $\mathrm{k}_{M}$ for some $M$. The smallest such $M$ is called the conductor of $F$. From now on we always assume that every extension of $\mathrm{k}$ is contained in some cyclotomic function field. Let $\ell$ be a prime number different from the characteristic of $\mathrm{k}$ and $r$ be the smallest positive integer such that $\ell \mid q^{r}-1$.

Let $F$ be a $\ell$-cyclic extension of $\mathrm{k}$, and write $N=N_{F}$ for the conductor of $F$. Then $N$ must be square-free since $F / \mathrm{k}$ is tamely ramified and for each prime divisor $P$ of $N$, deg $P$ is divisible by $r$, since the ramification index $\ell$ 
divides $q^{\operatorname{deg} P}-1$, the order of the multiplicative group of the residue field of $P$. Write $N=P_{1} \cdots P_{t}$. It is easy to see that the number of such extensions $F$ with conductor $P_{1} \cdots P_{t}$ is $(\ell-1)^{t-1}$. Write $\mathrm{H}_{F}$ for the Hilbert class field of $F$. Then the genus field $\mathrm{G}_{F}$ of $F / \mathrm{k}$ is defined to be the maximal extension of $F$ in $\mathrm{H}_{F}$ which is the compositum of $F$ and some abelian extension of k. Let $\mathcal{C l}(F)$ be the ideal class group of the integral closure $\mathcal{O}_{F}$ of $\mathbb{A}$ in $F$, and $\mathcal{C l}(F)_{\ell}$ be its Sylow $\ell$-subgroup. Let $\sigma$ be a fixed generator of $G=\operatorname{Gal}(F / \mathrm{k})$ and

$$
\lambda_{i}(F):=\operatorname{dim}_{\mathbb{F}_{\ell}}\left(\mathcal{C l}(F)_{\ell}^{(\sigma-1)^{i-1}} / \mathcal{C l}(F)_{\ell}^{(\sigma-1)^{i}}\right) \text { for } i \geq 1 .
$$

It is known that $([2, \S 2])$

$$
\mathcal{C l}(F)_{\ell} / \mathcal{C l}(F)_{\ell}^{\sigma-1} \simeq \mathcal{C l}(F) / \mathcal{C l}(F)^{\sigma-1} \simeq \mathrm{Gal}\left(\mathrm{G}_{F} / F\right) .
$$

It is well-known that $\mathcal{C l}(F)_{\ell}^{G}$ and $\mathcal{C l}(F)_{\ell} / \mathcal{C l}(F)_{\ell}^{\sigma-1}$ are elementary abelian groups of rank $\lambda_{1}$. Since $F$ is contained in some cyclotomic function field, the inertia degree $f_{\infty}$ at $\infty$ should be 1 , and the ramification degree $e_{\infty}$ is 1 if $r>1$.

Now we consider the narrow case. We define the narrow Hilbert class field $\mathrm{H}_{F}^{+}$of $F$ to be the maximal abelian extension of $F$ in $\Omega$, unramified outside the places over $\infty$. For each place $v$ of $F$ over $\infty$ we write $F_{v}$ to denote the completion of $F$ at $v$ and $N_{v}$ be the norm map from $F_{v}$ to $\mathrm{k}_{\infty}$. We define a $\operatorname{sign} \operatorname{map} \operatorname{sgn}_{v}: F_{v} \rightarrow \mathbb{F}_{q}$ by $\operatorname{sgn}_{v}(x)=\operatorname{sgn}\left(N_{v}(x)\right)$, where $\operatorname{sgn}$ is the usual sign map on $\mathrm{k}_{\infty}$. An element $x \in F$ is called totally positive if $\operatorname{sgn}_{v}(x)=1$ for any $v$ lying over $\infty$. Denote by $F_{+}$the set of all totally positive elements of $F$. The narrow ideal class group $\mathrm{Cl}^{+}(F)$ of $F$ is defined to be the quotient group of fractional ideals modulo principal fractional ideals generated by elements of $F_{+}$. The narrow genus field $\mathrm{G}_{F}^{+}$of $F / \mathrm{k}$ is defined to be the maximal extension of $F$ in $\mathrm{H}_{F}^{+}$which is the compositum of $F$ and some abelian extension of $\mathrm{k}$. See [2] for details on the genus theory of function fields. Let

$$
\lambda_{i}^{+}(F):=\operatorname{dim}_{\mathbb{F}_{\ell}}\left(\mathcal{C} l^{+}(F)_{\ell}^{(\sigma-1)^{i-1}} / \mathcal{C} l^{+}(F)_{\ell}^{(\sigma-1)^{i}}\right) \text { for } i \geq 1 .
$$

Note that if $r>1$, then $\mathcal{C} l^{+}(F)_{\ell}=\mathcal{C l}(F)_{\ell}$ and so $\lambda_{i}^{+}(F)=\lambda_{i}(F)$. We will use the following lemmas proved in [13]. The narrow case can be proved by a similar method as in [13].

Lemma 2.1 ([13, Theorem 2.1]). Let $F$ be as above.

(i) If $r>1$, or $r=1$ and $\ell \mid \operatorname{deg} P_{i}$ for all $i$, then $\lambda_{1}(F)=t-1$.

(ii) In all other cases, $\lambda_{1}(F)=t-2+\log _{\ell}\left(e_{\infty} f_{\infty}\right)$.

(iii) $\lambda_{1}^{+}(F)=t-1$.

Let $\mathfrak{p}_{i}$ be the unique prime ideal of $F$ lying above $P_{i}$.

Lemma 2.2 ([13, Corollary 2.3, 2.4]). Let $F$ be as above.

(i) If $r>1$, then $\mathcal{C l}(F)_{\ell}^{G}$ is generated by the classes $\left[\mathfrak{p}_{1}\right], \ldots,\left[\mathfrak{p}_{t}\right]$. 
(ii) If $r=1$, then

$$
\mathcal{C l}(F)_{\ell}^{G}=\left\langle\left[\mathfrak{p}_{1}\right], \ldots,\left[\mathfrak{p}_{t}\right]\right\rangle,
$$

except the case that $\ell \mid \operatorname{deg} P_{i}$ for all $i$ and $N_{F / \mathrm{k}}\left(\mathcal{O}_{F}^{*}\right)=\left(\mathbb{F}_{q}^{*}\right)^{\ell}$. In this case,

$$
\mathcal{C l}(F)_{\ell}^{G}=\left\langle\left[\mathfrak{p}_{1}\right], \ldots,\left[\mathfrak{p}_{t}\right],[\mathfrak{a}]\right\rangle,
$$

where $\mathfrak{a}^{\sigma-1}=\alpha \mathcal{O}_{F}$ and $N_{F / \mathrm{k}}(\alpha) \in \mathbb{F}_{q}^{*} \backslash\left(\mathbb{F}_{q}^{*}\right)^{\ell}$.

(iii) If $r=1$ and $\infty$ splits completely, then $\mathcal{C l} l^{+}(F)_{\ell}^{G}$ is generated by $\left[\mathfrak{p}_{1}\right]_{+}$, $\ldots,\left[\mathfrak{p}_{t}\right]_{+}$and $[\mathfrak{a}]_{+}$, where $\mathfrak{a}^{\sigma-1}=\alpha \mathcal{O}_{F}$ and $N_{F / \mathrm{k}}(\alpha) \in \mathbb{F}_{q}^{* \ell} \backslash \mathbb{F}_{q}^{* \ell^{2}}$. In particular, if $\ell \|(q-1)$, then $\mathrm{Cl}^{+}(F)_{\ell}^{G}$ is generated by $\left[\mathfrak{p}_{1}\right]_{+}, \ldots,\left[\mathfrak{p}_{t}\right]_{+}$.

Proof. We only need to prove (iii). Recall that a fractional ideal $\mathfrak{a}$ of $\mathcal{O}_{F}$ is said to be ambiguous if it is invariant under the $G$-action, i.e., $\mathfrak{a}^{\sigma}=\mathfrak{a}$. Any ideal class in $\mathcal{C l}(F)^{G}$ or $\mathcal{C l} l^{+}(F)^{G}$ is called an ambiguous ideal class. Let $\mathfrak{a}$ be an ideal representing an ambiguous ideal class. Then $\mathfrak{a}^{\sigma-1}=\alpha \mathcal{O}_{F}$ with $\alpha \in F_{+}$. Then $N_{F / \mathrm{k}}(\alpha) \in \mathbb{F}_{q}^{* \ell}$, that is, $N_{F / \mathrm{k}}(\alpha)=\eta^{-\ell}$, and so $N_{F / \mathrm{k}}(\alpha \eta)=1$ for some $\eta \in \mathbb{F}_{q}^{*}$. By Hilbert Theorem 90, there exists $\beta \in F$ such that $\alpha \eta=\beta^{\sigma} / \beta$. Then, since

$$
\mathfrak{a}^{\sigma-1}=(\alpha \eta)=\left(\beta^{\sigma} / \beta\right)
$$

$\beta^{-1} \mathfrak{a}$ is an ambiguous ideal. Therefore, $[\mathfrak{a}]_{+} \in\left\langle\left[\mathfrak{p}_{1}\right]_{+}, \ldots,\left[\mathfrak{p}_{t}\right]_{+}\right\rangle$if and only if $\beta \in F_{+}$, which is equivalent to $\alpha \eta \in F_{+}$. But since $\alpha \in F_{+}$, this is equivalent to $\eta \in \mathbb{F}_{q}^{* \ell}$.

We know from $[13, \S 2]$ that

$$
\left\langle\left[\mathfrak{p}_{1}\right], \ldots,\left[\mathfrak{p}_{t}\right]\right\rangle=I(F)^{G} P(F) / P(F)=I(F)^{G} / I(F)^{G} \cap P(F)
$$

has $\ell$-rank at least $t-2$. Thus $\left\langle\left[\mathfrak{p}_{1}\right]_{+}, \ldots,\left[\mathfrak{p}_{t}\right]_{+}\right\rangle=I(F)^{G} P\left(F_{+}\right) / P\left(F_{+}\right)=$ $I(F)^{G} / I(F)^{G} \cap P\left(F_{+}\right)$has $\ell$-rank at least $t-2$. It is not hard to see that the $\ell$-rank of $\mathrm{Cl}^{+}(F)_{\ell}^{G}$ is $t-1$, thus we get the result.

Remark 2.3. In the number field case, since the size of the group of units in $\mathbb{Z}$ is 2 , there does not exist such an ideal $\mathfrak{a}$ as in Lemma 2.2(iii).

Suppose first that $r=1$. In this case $F=\mathrm{k}(\sqrt[\ell]{D})$, where $D=a P_{1}^{e_{1}} \cdots P_{t}^{e_{t}}$ with $1 \leq e_{i}<\ell$ and $a \in \mathbb{F}_{q}^{*}$. We will determine $a$. From [1, Lemma 3.2], it is known that if $\ell \mid \operatorname{deg} P_{i}$, then $\mathrm{k}\left(\sqrt[\ell]{P_{i}}\right) \subseteq \mathrm{k}_{P_{i}}$, and that if $\ell \nmid \operatorname{deg} P_{i}$, then $\mathrm{k}\left(\sqrt[\ell]{-P_{i}^{d_{i}}}\right) \subseteq \mathrm{k}_{P_{i}}$, where $d_{i}$ is a positive integer such that $d_{i} \operatorname{deg} P_{i} \equiv 1 \bmod \ell$. Thus we see that $a$ can be taken to be $(-1)^{m}$, where $m=\sum_{\ell \nmid \operatorname{deg} P_{i}} \nu_{i}$ and $d_{i} \nu_{i} \equiv e_{i} \bmod \ell$. When $\ell \neq 2$, or $q \equiv 1 \bmod 4$ and $\ell=2,-1$ is an $\ell$-th power in $\mathbb{F}_{q}^{*}$. Thus one may take $a$ to be 1 in these cases. If $q \equiv 3 \bmod 4$ and $\ell=2$, then we take $a=(-1)^{s}$, where $s$ is the number of odd degree $P_{i}$ 's.

Proposition 2.4 ([13, Theorem 2.5]). Let $F=\mathrm{k}(\sqrt[\ell]{D})$ be as above.

(i) $\mathrm{G}_{F}^{+}=\mathrm{k}\left(\sqrt[\ell]{(-1)^{\operatorname{deg} P_{1} P_{1}}}, \ldots, \sqrt[\ell]{(-1)^{\operatorname{deg} P_{t} P_{t}}}\right)$. 
(ii) If $\ell \nmid \operatorname{deg} D$ or $\ell \mid \operatorname{deg} P_{i}$ for all $i$, then

$$
\mathrm{G}_{F}=\mathrm{G}_{F}^{+}=\mathrm{k}\left(\sqrt[\ell]{(-1)^{\operatorname{deg} P_{i} P_{1}}}, \ldots, \sqrt[\ell]{(-1)^{\operatorname{deg} P_{t} P_{t}}}\right) .
$$

(iii) If $\ell \mid \operatorname{deg} D$ but $\ell \nmid \operatorname{deg} P_{i}$ for $1 \leq i \leq s$ and $\ell \mid \operatorname{deg} P_{j}$ for $s+1 \leq j \leq t$, then

$$
\mathrm{G}_{F}=\mathrm{k}\left(\sqrt[\ell]{P_{1} P_{2}^{u_{2}}}, \ldots, \sqrt[\ell]{P_{1} P_{s}^{u_{s}}}, \sqrt[\ell]{P_{s+1}}, \ldots, \sqrt[\ell]{P_{t}}\right)
$$

where $\operatorname{deg} P_{1}+u_{i} \operatorname{deg} P_{i} \equiv 0 \bmod \ell$.

Let $\eta$ be a fixed primitive $\ell$-th root of unity in $\mathbb{F}_{q}$. Let $\left(\frac{A}{P}\right)_{\ell}$ be the $\ell$-th power residue symbol. For a field $F$ as above, we define a $t \times t$ matrix $M_{F}=\left(m_{i j}\right)$ over $\mathbb{F}_{\ell}$ by, for $i \neq j$,

$$
\eta^{m_{i j}}=\left(\frac{\bar{P}_{i}}{P_{j}}\right)_{\ell}
$$

where $\bar{P}_{i}=(-1)^{\operatorname{deg} P_{i}} P_{i}$ and $m_{i i}$ is defined to satisfy

$$
\sum_{i=1}^{t} e_{i} m_{i j}=0 .
$$

If $\mathfrak{a}$ is as in Lemma $2.2(\mathrm{ii})$ or (iii), let $N_{F / \mathrm{k}}(\mathfrak{a})=(A)$ for some $A \in \mathbb{A}$. Then $m_{i 0}$ is defined to be

$$
\eta^{m_{i 0}}=\left(\frac{P_{i}}{A}\right)_{\ell}
$$

Let $M_{F}:=\left(m_{i j}\right)$, which is a $t \times t$ or $t \times(t+1)$ matrix with entries in $\mathbb{F}_{\ell}$ according to the existence of $\mathfrak{a}$ as in Lemma 2.2. Then it can be shown (cf. $[13, \S 3])$ that

$$
\lambda_{2}(F)=\lambda_{1}(F)-\operatorname{rank}\left(M_{F}\right), \text { when } \infty \text { ramifies in } F
$$

and

$$
\lambda_{2}^{+}(F)=\lambda_{1}^{+}(F)-\operatorname{rank}\left(M_{F}\right) \text {, when } \infty \text { splits in } F .
$$

In the case (iii) of Proposition 2.4, a $(t-1) \times t$ matrix $M_{F}^{\prime}$ is defined in [13, $\S 3]$ and it was shown that

$$
\lambda_{2}(F)=t-2-\operatorname{rank}\left(M_{F}^{\prime}\right) .
$$

Now suppose that $r>1$. Let

$$
w=\sum_{i=1}^{t}\left(\operatorname{deg} P_{i}, r\right),
$$

where $(a, b)$ denotes the greatest common divisor of $a$ and $b$. A $t \times w$ matrix $\tilde{M}_{F}$ over $\mathbb{F}_{\ell}$ is defined in $[13, \S 4]$ and it is shown that

$$
\lambda_{2}(F)=t-1-\operatorname{rank}\left(\tilde{M}_{F}\right) .
$$


Let $\mathrm{k}^{\prime}=\mathbb{F}_{q^{r}} \cdot \mathrm{k}$ and $F^{\prime}=\mathbb{F}_{q^{r}} \cdot F$. Let the notations be as in [13, §4]. By identifying $\operatorname{Gal}\left(F^{\prime} / \mathrm{k}^{\prime}\right) \cong \operatorname{Gal}(F / \mathrm{k}) \cong \mathbb{F}_{\ell}$, we have

$$
\left(\frac{Q_{i}}{P_{j}}\right)_{\ell}=\left(\frac{Q_{i}, F^{\prime} / \mathrm{k}^{\prime}}{\left(P_{j}\right)}\right)
$$

and

$$
\left.\left(\frac{Q_{i}, F^{\prime} / \mathrm{k}^{\prime}}{\left(P_{j}\right)}\right)\right|_{F}=\left(\frac{P_{i}, F / \mathrm{k}}{\left(P_{j}\right)}\right) .
$$

Thus the matrix $\tilde{M}_{F}$ is essentially the same as the matrix $M_{3}$ defined in [6, $\S 2]$. We let $M_{F}$ be $M_{3}$. The product formula for norm residue symbol implies that the sum of each column of $M_{F}$ is zero.

\section{Asymptotic behavior of $\ell$-cyclic extensions with prescribed $\ell$-class numbers}

Let $F$ be a cyclic extension of k of degree $\ell$ with conductor $N=P_{1} \cdots P_{s}$. Let $\mathrm{H}_{i}$ be the unique cyclic extension of $\mathrm{k}$ of degree $\ell$ with conductor $P_{i}$. Let $\mathrm{K}_{i}=F \cdot \mathrm{H}_{i}$ and $\mathrm{K}=\mathrm{K}_{1} \cdots \mathrm{K}_{t}$.

\section{1. $r>1$ case}

In this subsection we assume that $r>1$. Let

- $N_{s, n}:=$ the number of $\ell$-cyclic extensions $F$ of $\mathrm{k}$ with $\left|\mathcal{C l}(F)_{\ell}\right|=\ell^{s}$ and with conductor $N$ of degree $n$,

- $M_{s, n}:=$ the number of $\ell$-cyclic extensions $F$ of k with $\left|\mathcal{C l}(F)_{\ell}\right|=\ell^{s-1}$ and with conductor $N$ of degree $n$ such that $N$ has exactly $s$ distinct prime factors,

- $G_{s, n}:=$ the number of $\ell$-cyclic extensions $F$ of $\mathrm{k}$ with conductor $N=$ $P_{1} \cdots P_{s}$ of degree $n$ such that $P_{m}$ is an $\ell$-th power residue modulo $P_{1}, \ldots, P_{m-2}$ but an $\ell$-th power nonresidue modulo $P_{m-1}$.

It can be shown, as in Theorem 1 of [4], that if $F$ is an $\ell$-cyclic extension of $\mathrm{k}$ satisfying the conditions to define $G_{s, n}$, then the $\ell$-class group of $F$ is an elementary abelian $\ell$-group of rank $s-1$. Hence $M_{s, n} \geq G_{s, n}$.

Since we know that $r$ must divide the degrees of prime factors of $N$, we replace $n$ by $r n$ and write $\operatorname{deg} P_{i}=r k_{i}$.

Let $\chi_{P_{i}}$ be a Dirichlet character of exponent $\ell$ of conductor $P_{i}$, that is, a character of $\operatorname{Gal}\left(\mathrm{k}_{P_{i}} / \mathrm{k}\right)$. For a prime $P_{m} \neq P_{1}, \ldots, P_{m-1}$, let

$$
W_{m}:=\frac{1}{\ell^{m-1}}\left(\sum_{j_{1}=0}^{\ell-1} \chi_{P_{1}}^{j_{1}}\left(P_{m}\right)\right) \cdots\left(\sum_{j_{m-2}=0}^{\ell-1} \chi_{P_{m-2}}^{j_{m-2}}\left(P_{m}\right)\right)\left(\sum_{j_{m-1}=0}^{\ell-1} \zeta^{j_{m-1}} \chi_{P_{m-1}}^{j_{m-1}}\left(P_{m}\right)\right),
$$

where $\zeta$ is a primitive $\ell$-th root of unity. Since $\sum_{k=0}^{\ell-1} \chi_{P_{j}}^{k}\left(P_{m}\right)=\ell$ or 0 , depending on $P_{m}$ is $\ell$-th power residue modulo $P_{j}$ or not, we have

$$
M_{t, r n} \geq G_{t, r n} \geq \sum W_{2} \cdots W_{t},
$$


where the sum is over the distinct primes $P_{1}, \ldots, P_{t}$ with $\operatorname{deg}\left(P_{1} \cdots P_{t}\right)=r n$ and $r \mid \operatorname{deg} P_{i}$. Let $y_{i}:=2^{i} \sqrt{n}$. Then $y_{1}+\cdots+y_{t-1}<y_{t}=y$. Let

$$
A_{t, r n}:=\sum W_{2} \cdots W_{t-1} \sum_{P_{t}, \operatorname{deg} P_{t}=r n-\operatorname{deg} P_{1}-\cdots-\operatorname{deg} P_{t-1}} W_{t},
$$

where the first sum is over distinct $P_{i}, 1 \leq i \leq t-1$ with $\operatorname{deg} P_{i-1} \leq \operatorname{deg} P_{i} \leq y_{i}$ and $r \mid \operatorname{deg} P_{i}$. Then

$$
M_{t, r n} \geq G_{t, r n} \geq A_{t, r n}
$$

Write

$$
W_{t}=\frac{1}{\ell^{t-1}}\left(1+\sum_{J} \zeta^{j_{t-1}} \chi_{P_{1}}^{j_{1}} \cdots \chi_{P_{t-1}}^{j_{t-1}}\left(P_{t}\right)\right)
$$

where $J=\left(j_{1}, \ldots, j_{t-1}\right) \neq(0, \ldots, 0)$. Then, by (1.1) and (1.5),

$\sum_{\operatorname{deg} P_{t}=r\left(n-k_{1}-\cdots-k_{t-1}\right)} W_{t}=\frac{q^{r\left(n-k_{1}-\cdots-k_{t-1}\right)}}{\ell^{t-1} r\left(n-k_{1}-\cdots-k_{t-1}\right)}+O\left(\frac{q^{r\left(n-k_{1}-\cdots-k_{t-1}\right) / 2}}{n-k_{1}-\cdots-k_{t-1}}\right)$.

For $k_{i} \leq y_{i}$, since $n-y=n-2^{t} \sqrt{n}>n / 2$ for large $n$,

$$
\begin{aligned}
& \frac{q^{r\left(n-k_{1}-\cdots-k_{t-1}\right)}}{r\left(n-k_{1}-\cdots-k_{t-1}\right)} \\
= & \frac{q^{r\left(n-k_{1}-\cdots-k_{t-1}\right)}}{r n}+\frac{q^{r\left(n-k_{1}-\cdots-k_{t-1}\right)}\left(k_{1}+\cdots+k_{t-1}\right)}{r n\left(n-k_{1}-\cdots-k_{t-1}\right)} \\
= & \frac{q^{r\left(n-k_{1}-\cdots-k_{t-1}\right)}}{r n}+O\left(\frac{\left(k_{1}+\cdots+k_{t-1}\right) q^{r\left(n-k_{1}-\cdots-k_{t-1}\right)}}{n^{2}}\right)
\end{aligned}
$$

and

$$
\frac{q^{r\left(n-k_{1}-\cdots-k_{t-1}\right) / 2}}{\left(n-k_{1}-\cdots-k_{t-1}\right)}=O\left(\frac{q^{r\left(n-k_{1}-\cdots-k_{t-1}\right)}}{n^{2}}\right)
$$

Thus

$$
\sum_{\operatorname{deg} P_{t}=r\left(n-k_{1}-\cdots-k_{t-1}\right)} W_{t}=\frac{q^{r\left(n-k_{1}-\cdots-k_{t-1}\right)}}{\ell^{t-1} r n}+O\left(\frac{\left(k_{1}+\cdots+k_{t-1}\right) q^{r\left(n-k_{1}-\cdots-k_{t-1}\right)}}{n^{2}}\right) .
$$

From (1.7) and (1.9) we have, for $y=y_{t}=2^{t} \sqrt{n}$,

$$
\sum_{\substack{P_{1}, \ldots, P_{t-1} \\ r \mid \operatorname{deg} P_{i} \leq r y_{i}}} \frac{q^{r n}\left(\operatorname{deg} P_{1}+\cdots+\operatorname{deg} P_{t-1}\right)}{n^{2} q^{\operatorname{deg} P_{1}} \cdots q^{\operatorname{deg} P_{t-1}}}=O\left(\frac{y(\log y)^{t-2} q^{r n}}{n^{2}}\right)=O\left(\frac{q^{r n}}{n}\right) .
$$

Therefore

$$
A_{t, r n}=\frac{1}{\ell^{t-1}} \sum_{\substack{P_{1}, \ldots, P_{t-1}: \text { distinct } \\ r \mid \operatorname{deg} P_{i} \leq r y_{i}}} W_{2} \cdots W_{t-1} \frac{q^{n r}}{r n q^{\operatorname{deg} P_{1}} \cdots q^{\operatorname{deg} P_{t-1}}}+O\left(\frac{q^{r n}}{n}\right) .
$$

Now

$$
W_{t-1}=\frac{1}{\ell^{t-2}}\left(1+\sum_{J} \zeta^{j_{t-1}} \chi_{P_{1}}^{j_{1}} \cdots \chi_{P_{t-2}}^{j_{t-2}}\left(P_{t-1}\right)\right)
$$


Let $\chi_{Q}$ be a nontrivial character with exponent $\ell$ and conductor $Q \mid P_{1} \cdots P_{t-2}$. Let

$$
S_{Q}(u):=\sum_{\operatorname{deg} P_{t-1}=r u} \chi_{Q}\left(P_{t-1}\right)
$$

Then, by (1.5)

$$
\sum_{u=\frac{\operatorname{deg} P_{t-2}}{r}}^{\left[y_{t-1}\right]} \frac{S_{Q}(u)}{q^{r u}}=\sum_{u=\frac{\operatorname{deg} P_{t-2}}{r}}^{\left[y_{t-1}\right]} O\left(\frac{1}{u q^{\frac{r u}{2}}}\right)=O(1) .
$$

Continuing the same process, we have

$$
A_{t, r n}=\frac{1}{\ell^{t(t-1) / 2}} \sum_{P_{1}, \ldots, P_{t-1}} \frac{q^{r n}}{r n q^{\operatorname{deg} P_{1}} \cdots q^{\operatorname{deg} P_{t-1}}}+O\left(\frac{q^{r n}}{n}(\log y)^{t-2}\right) .
$$

Thus

$$
A_{t, r n}=c \frac{q^{r n}(\log n)^{t-1}}{r n}+O\left(\frac{q^{r n}(\log n)^{t-2}}{n}\right) .
$$

Since $M_{s, r n}=O\left(p_{r}(r n, s)\right)$, we have, from (1.4) and (3.2),

$$
M_{s, r n}=O\left(\frac{q^{r n}}{n}(\log n)^{s-1}\right) .
$$

If an $\ell$-extension $F$ of $\mathrm{k}$ has $\left|\mathcal{C} l(F)_{\ell}\right|=\ell^{s}$, then at most $s+1$ primes can be ramified. Now the rest of the argument to prove Theorem 1 in [5] works here and we get

\section{Theorem 3.1.}

$$
N_{s, r n}=M_{s+1, r n}+O\left(\frac{M_{s+1, r n}}{\log n}\right) .
$$

We will compute $M_{s+1, r n}$. As in $[6, \S 2, \S 3]$, one can see easily that the $\ell$-cyclic extension $F$ has $\ell$-class number $\ell^{s}$ precisely when $\operatorname{rank}\left(M_{F}\right)=s$, and that the number of distinct $(s+1) \times(s+1)$ matrices $\Gamma$ over $\mathbb{F}_{\ell}$ such that $\operatorname{rank}(\Gamma)=s$ and such that $\Gamma=M_{F}$ for some field $F$ is

$$
\ell^{\frac{s(s-1)}{2}}(\ell-1)^{s} \prod_{i=1}^{s}\left(\ell^{i}+\cdots+\ell+1\right) .
$$

Note that $\Gamma=M_{F}$ if and only if the sum of each column of $\Gamma$ is 0 by the product formula of Hilbert symbols.

Now we consider the number $N(\Gamma)$ of $F$ with conductor $N=P_{1} \cdots P_{s+1}$ of degree $r n$ and the corresponding matrix $M_{F}=\Gamma$. Let $\mathrm{k}^{\prime}=\mathbb{F}_{q^{r}} \cdot \mathrm{k}$ and $\mathrm{L}_{i}=$ $\mathrm{k}^{\prime} \cdot \mathrm{H}_{i}$, where $\mathrm{H}_{i}$ is the $\ell$-cyclic extension of $\mathrm{k}$ with conductor $P_{i}$. Then $\mathrm{L}_{i} / \mathrm{k}^{\prime}$ is a Kummer extension $\mathrm{L}_{i}=\mathrm{k}^{\prime}\left(\sqrt[\ell]{\mu_{i}}\right)$ for some $\mu_{i} \in \mathrm{k}^{\prime}$. Then $\mathrm{k} \cdot F=\mathrm{k}^{\prime}(\sqrt[\ell]{\mu})$ with $\mu=\mu_{1}^{e_{1}} \cdots \mu_{s+1}^{e_{s+1}}$. Let $\mathrm{L}_{i}^{\prime}=\mathrm{k}^{\prime}\left(\sqrt[\ell]{P_{i}}\right)$. Define, for a prime $P$ of degree divisible by $r, \lambda_{i}\left(\mathfrak{p}_{j}\right)$ and $\omega_{i}\left(\mathfrak{p}_{j}\right)$ as follows;

$$
\left(\mathfrak{p}_{j}, \mathrm{~L}_{i} / \mathrm{k}^{\prime}\right)\left(\sqrt[\ell]{\mu_{i}}\right)=\lambda_{i}\left(\mathfrak{p}_{j}\right)^{-1} \sqrt[\ell]{\mu_{i}}, \quad\left(\mathfrak{p}_{j}, \mathrm{~L}_{i}^{\prime} / \mathrm{k}^{\prime}\right)\left(\sqrt[\ell]{P_{i}}\right)=\omega_{i}\left(\mathfrak{p}_{j}\right)^{-1} \sqrt[\ell]{P_{i}},
$$


where $\mathfrak{p}$ is a prime of $\mathrm{k}^{\prime}$ lying over $P$. Let $\delta_{i}(j, k ; u, v)(P)=1$ if $\left(\lambda_{i}(P)^{j}, \omega_{i}(P)^{k}\right)$ $=\left(\zeta^{u}, \zeta^{v}\right)$, and 0 otherwise.

Lemma 3.2. We have, for $j=1, \ldots, \ell-1$,

$$
\sum_{\operatorname{deg} P=r m} \sum_{k=1}^{\ell-1} \delta_{i}(j, k ; u, v)(P) \sim \frac{\ell-1}{\ell^{2}} \frac{q^{r m}}{r m} .
$$

Proof. We may assume $j=1$. The probability for a prime $P$ to satisfy $\left(\lambda_{i}(P), \omega_{i}(P)\right)=\left(\zeta^{u}, \zeta^{v}\right)$ is $\frac{1}{\ell^{2}}$. When $v=0$, then $\omega_{i}(P)^{k}=1=\zeta^{0}$ for any $k=$ $1, \ldots, \ell-1$. Hence the probability for a prime $P$ to satisfy $\left(\lambda_{i}(P), \omega_{i}(P)^{k}\right)=$ $\left(\zeta^{u}, \zeta^{0}\right)$ for some $k=1, \ldots, \ell-1$ is $\frac{\ell-1}{\ell^{2}}$. When $v \neq 0$, then, for $\omega_{i}(P) \neq 1$, there exists a unique $k=1, \ldots, \ell-1$ such that $\omega_{i}(P)^{k}=\zeta^{v}$. Thus the probability for a prime $P$ to satisfy $\left(\lambda_{i}(P), \omega_{i}(P)^{k}\right)=\left(\zeta^{u}, \zeta^{v}\right)$ for some $k=1, \ldots, \ell-1$ is again $\frac{\ell-1}{\ell^{2}}$. Then the result follows from the equation (1.1).

Note the difference of (3.4) from the formula in [6, p. 200]. In the classical case the condition $p \equiv 1 \bmod \ell$ is imposed instead of the condition that $\operatorname{deg} P_{i}$ is divisible by $r$, and the probability for a prime to satisfy $p \equiv 1 \bmod \ell$ is $\frac{1}{\ell-1}$ by Dirichlet theorem on arithmetic progression. We repeat the process to get

$$
\sum_{\operatorname{deg}} \sum_{P_{w}=r m}^{\ell-1} \prod_{i=1}^{w-1} \delta_{i}(j, k ; u, v)\left(P_{w}\right) \sim \frac{\ell-1}{\ell^{2(w-1)}} \frac{q^{r m}}{r m} .
$$

Theorem 3.3. We have, for $(s+1) \times(s+1)$ matrix $\Gamma$ such that the sum of each column is 0 ,

and so

$$
N(\Gamma) \sim \frac{(\ell-1)^{s}}{s ! r^{s+1} \ell^{s^{2}+s}} \frac{q^{r n}(\log n)^{s}}{n},
$$

$$
N_{s, r n} \sim \frac{(\ell-1)^{2 s} \prod_{i=1}^{s}\left(\ell^{i}+\cdots+\ell+1\right)}{s ! r^{s+1} \ell^{\left(s^{2}+3 s\right) / 2}} \frac{q^{r n}(\log n)^{s}}{n} .
$$

Proof. (Sketch of proof) As in $\S 4$ of [6], we have

$N(\Gamma)=\frac{1}{(s+1) !} \sum_{m_{1}+\cdots+m_{s+1}=n} \sum_{\operatorname{deg}} \sum_{P_{1}=r m_{1} \operatorname{deg}} Y_{P_{2}=r m_{2}} \ldots \sum_{\operatorname{deg} P_{s}=r m_{s}} Y_{s} \sum_{\operatorname{deg}} \sum_{s+1} Y_{s+1}$,

where

$$
Y_{w}=\sum_{k=1}^{\ell-1} \prod_{i=1}^{w-1} \delta_{i}(j, k ; u, v)\left(P_{w}\right)
$$

for $w=2, \ldots, s+1$. Then using the equation (3.5), we have

$$
\begin{aligned}
N(\Gamma) & \sim \frac{1}{(s+1) !} \frac{(\ell-1)^{s}}{\ell^{s^{2}+s}} \frac{q^{r n}}{r^{s+1}} \sum_{m_{1}+\cdots+m_{s+1}=n} \frac{1}{m_{1} \cdots m_{s+1}} \\
& \sim \frac{(\ell-1)^{s}}{s ! r^{s+1} \ell^{s^{2}+s}} \frac{q^{r n}(\log n)^{s}}{n},
\end{aligned}
$$

by the equation (1.12). 
Remark 3.4. (1) One can do the error estimate as in [6] using (1.5) to get the error term $o\left(\frac{q^{r n}(\log n)^{s}}{n}\right)$. But it is not necessary for our purpose.

(2) In the proof of [6, Lemma 3], also in our proof here, the condition that $\Gamma$ has rank $s$ is not necessary to get the estimate $N(\Gamma)$. Thus the asymptotic formula for $N(\Gamma)$ works for any $\Gamma$ such that the sum of each column is 0 . This will be used in the computation in $\S 4$.

\section{2. $r=1$ case}

Now we assume that $r=1$, that is $\ell \mid q-1$. We consider $\ell$-cyclic extensions $F$ of $\mathrm{k}$ with conductor $N$ of degree $n$ and with $\left|\mathcal{C l}(F)_{\ell}\right|=\ell^{s}$. We have two cases. One is real, that is, $\infty$ splits completely. The other is imaginary, that is, $\infty$ ramifies. The case that $\infty$ is inert cannot happen, since we have assumed that the field is contained in some cyclotomic function field. Let

- $N_{I, s, n}:=$ the number of imaginary $\ell$-cyclic extensions $F$ of $\mathrm{k}$ with conductor $N$ of degree $n$ and $\left|\mathcal{C l}(F)_{\ell}\right|=\ell^{s}$,

- $N_{R, s, n}:=$ the number of real $\ell$-cyclic extensions $F$ of $\mathrm{k}$ with conductor $N$ of degree $n$ and $\left|\mathcal{C l}(F)_{\ell}\right|=\ell^{s}$,

- $M_{I, t, n}:=$ the number of imaginary $\ell$-cyclic extensions $F$ of $\mathrm{k}$ with conductor $N$ of degree $n$ such that $N$ has exactly $t$ distinct prime factors and $\left|\mathcal{C l}(F)_{\ell}\right|=\ell^{t-1}$,

- $M_{R, t, n}:=$ the number of real $\ell$-cyclic extensions $F$ of $\mathrm{k}$ with conductor $N$ of degree $n$ such that $N$ has exactly $t$ distinct prime factors and $\left|\mathcal{C l}(F)_{\ell}\right|=\ell^{t-2}$.

- For $\ell \mid n, M_{t, n}^{\prime}:=$ the number of $\ell$-cyclic extensions $F$ of conductor $N$ of degree $n$, such that $N$ has exactly $t$ prime factors, all having degrees divisible by $\ell$ and $\left|\mathcal{C l}(F)_{\ell}\right|=\ell^{t-1}$.

In this case $F=\mathrm{k}(\sqrt[\ell]{D})$ with $D=\alpha P_{1}^{e_{1}} \cdots P_{t}^{e_{t}}, 1 \leq e_{i} \leq l-1$. We may assume that $e_{1}=1$. Here $\alpha \in \mathbb{F}_{q}^{*}$ is chosen so that $F \subseteq \mathrm{k}_{N}$, where $N=P_{1} \cdots P_{t}$. If $\ell$ divides $\operatorname{deg} D$, then it is real. If $\ell$ does not divide $\operatorname{deg} D$, then it is imaginary. If $\ell=2$, then $\left(e_{1}, \ldots, e_{t}\right)=(1, \ldots, 1)$. In this case whether $F$ is real or imaginary depends only on the parity of $\operatorname{deg} N$. One can follow almost the same process to prove Theorem 3.1 as in the case $r>1$ to get

$$
N_{I, s, n}=M_{I, s+1, n}+O\left(\frac{M_{I, s+1, n}}{\log n}\right)
$$

and

$$
N_{R, s, n}= \begin{cases}M_{R, s+2, n}+O\left(\frac{M_{R, s+2, n}}{\log n}\right) & \text { for } \ell \nmid n, \\ M_{R, s+2, n}+M_{s+1, n}^{\prime}+O\left(\frac{M_{R, s+2, n}}{\log n}\right)+O\left(\frac{M_{s+1, n}^{\prime}}{\log n}\right) & \text { for } \ell \mid n .\end{cases}
$$


But one can show as in the case $r>1$ that $M_{R, s+2, n} \sim c \frac{q^{n}(\log n)^{s+1}}{n}$ for some $c>0$ and $M_{s+1, n}^{\prime}=O\left(\frac{q^{n}(\log n)^{s}}{n}\right)$. Therefore,

$$
N_{R, s, n}=M_{R, s+2, n}+O\left(\frac{M_{R, s+2, n}}{\log n}\right) .
$$

Some calculation concerning this will be carried out in $\S 4.2$, where we replace $\mathcal{C l}(F)$ by $\mathrm{Cl}^{+}(F)$.

Remark 3.5. From the equations (1.2) and (1.3), it is very likely that $M_{s+1, n}^{\prime}=$ $o\left(M_{R, s+2, n}\right)$.

\section{Density for $\ell$-ranks of $\ell$-cyclic function fields}

\section{1. $r>1$ case}

In this subsection we assume $r>1$, that is $\ell \nmid q-1$. Let $\mathbf{A}_{t}$ be the set of all $\ell$-cyclic extensions $F$ of $\mathrm{k}$ such that $t$ finite primes ramify in $F / \mathrm{k}$, and

$$
\begin{aligned}
& \mathbf{A}_{t ; n}:=\left\{F \in \mathbf{A}_{t} \mid \operatorname{deg}(\operatorname{cond}(F))=n\right\}, \\
& \mathbf{A}_{t, e}:=\left\{F \in \mathbf{A}_{t} \mid \lambda_{2}(F)=e\right\}, \\
& \mathbf{A}_{t, e ; n}:=\mathbf{A}_{t, e} \cap \mathbf{A}_{t ; n},
\end{aligned}
$$

where $\operatorname{cond}(F)$ denotes the conductor of $F$. We define the density $d_{t, e}$ by

$$
d_{t, e}:=\lim _{n \rightarrow \infty} \frac{\left|\mathbf{A}_{t, e ; r n}\right|}{\left|\mathbf{A}_{t ; r n}\right|} .
$$

Note that $\mathbf{A}_{t, 0 ; n}=M_{t, n}$ in $\S 3.1$. For any monic irreducible polynomials $P_{1}, \ldots, P_{t}$ with $r \mid \operatorname{deg} P_{i}$, there are $(\ell-1)^{t-1}$ distinct fields $F$ in $\mathbf{A}_{t}$ with conductor $N=P_{1} \cdots P_{t}$. So by (1.3), we have

$$
\left|\mathbf{A}_{t ; r n}\right|=(\ell-1)^{t-1} \sum_{\substack{\operatorname{deg}\left(P_{1} \cdots P_{t}\right)=r n \\ r \mid \operatorname{deg} P_{i}}} 1 \sim \frac{(\ell-1)^{t-1}}{(t-1) ! r^{t}} \frac{q^{r n}(\log n)^{t-1}}{n} .
$$

Let $M_{F}$ be the $t \times t$ matrix over $\mathbb{F}_{\ell}$ associated to $F$ as in $\S 2$. Following the arguments in $[6, \S 2, \S 3]$, we see that $\lambda_{2}(F)=t-1-\operatorname{rank}\left(M_{F}\right)$. Then $\left|\mathbf{A}_{t, e ; r n}\right|$ can be estimated as

$$
\left|\mathbf{A}_{t, e ; r n}\right| \sim \sum_{\substack{\Gamma \\ \operatorname{rank}(\Gamma)=t-1-e}} \sum_{\substack{\operatorname{deg}\left(P_{1} \cdots P_{t}\right)=r n \\ r \mid \operatorname{deg} P_{i}}} \sum_{\substack{F \\ \operatorname{cond}(F)=P_{1} \cdots P_{t}}} \delta_{\Gamma, F},
$$

where $\delta_{\Gamma, F}=1$ if $M_{F}=\Gamma$ and $\delta_{\Gamma, F}=0$ otherwise. It is shown in Theorem 3.3 that, for $t \times t$ matrix $\Gamma$ such that the sum of each column is 0 ,

$$
N(\Gamma)=\sum_{\substack{\operatorname{deg}\left(P_{1} \ldots P_{t}\right)=r n \\ r \mid \operatorname{deg} P_{i}}} \sum_{\substack{F \\ \operatorname{cond}(F)=P_{1} \cdots P_{t}}} \delta_{\Gamma, F} \sim \frac{(\ell-1)^{t-1}}{(t-1) ! r^{t} \ell^{t(t-1)}} \frac{q^{r n}(\log n)^{t-1}}{n} .
$$


It is known ([7, Proposition 2.1]) that the number $N(t, t-1-e)$ of $t \times t$ matrices $\Gamma$, where the sum of each column is 0 , over $\mathbb{F}_{\ell}$ with rank $t-1-e$ is

$$
N(t, t-1-e)=\left[\prod_{j=1}^{t-1-e}\left(\ell^{t}-\ell^{j-1}\right)\right] \sum_{\substack{k_{1}+\cdots+k_{t-1-e} \leq e \\ \text { each } k_{i} \geq 0}}\left(\prod_{s=1}^{t-1-e} \ell^{s k_{s}}\right) .
$$

So we have, from (4.2), (4.3) and (4.4),

$$
\left|\mathbf{A}_{t, e ; r n}\right| \sim \frac{(\ell-1)^{t-1}}{(t-1) ! r^{t} \ell^{t(t-1)}} \frac{q^{r n}(\log n)^{t-1}}{n}\left[\prod_{j=1}^{t-1-e}\left(\ell^{t}-\ell^{j-1}\right)\right] \sum_{\substack{k_{1}+\ldots+k_{t-1-e} \leq e \\ \text { each } k_{i} \geq 0}}\left(\prod_{s=1}^{t-1-e} \ell^{s k_{s}}\right) .
$$

Thus

$$
d_{t, e}=\frac{1}{\ell^{t e}}\left[\prod_{j=1}^{t-1-e}\left(1-\frac{1}{\ell^{t+1-j}}\right)\right] \sum_{\substack{k_{1}+\cdots+k_{t-1-e} \leq e \\ \text { each } k_{i} \geq 0}}\left(\prod_{s=1}^{t-1-e} \ell^{s k_{s}}\right)
$$

for $1 \leq e \leq t-1$ and $d_{t, t-1}=\ell^{-t(t-1)}$. Let

$$
d_{\infty, e}:=\lim _{t \rightarrow \infty} d_{t, e}
$$

Then we follow almost the same argument as in $[10, \S 3]$ to get

$$
d_{\infty, e}=\frac{\ell^{-e(e+1)} \prod_{k=1}^{\infty}\left(1-\ell^{-k}\right)}{\prod_{k=1}^{e}\left(1-\ell^{-k}\right) \prod_{k=1}^{e+1}\left(1-\ell^{-k}\right)} \quad \text { for } e=0,1,2, \ldots
$$

\section{2. $r=1$ case}

Let $\mathbf{A}_{t}$ be the set of all $\ell$-cyclic extensions $F$ such that $t$ finite primes ramify in $F / \mathrm{k}$, and

$$
\begin{aligned}
& \mathbf{A}_{t ; n}:=\left\{F \in \mathbf{A}_{t} \mid \operatorname{deg}(\operatorname{cond}(F))=n\right\}, \\
& \mathbf{A}_{t, e}:=\left\{F \in \mathbf{A}_{t} \mid \lambda_{2}(F)^{+}=e\right\}, \\
& \mathbf{A}_{t, e ; n}:=\mathbf{A}_{t, e} \cap \mathbf{A}_{t ; n},
\end{aligned}
$$

where $\operatorname{cond}(F)$ denotes the conductor of $F$. We define the density $d_{t, e}$ by

$$
d_{t, e}:=\lim _{n \rightarrow \infty} \frac{\left|\mathbf{A}_{t, e ; n}\right|}{\left|\mathbf{A}_{t ; n}\right|} .
$$

For each $N \in P(n, t)$ and $\mathbf{e}:=\left(1, e_{1}, \ldots, e_{t}\right)$ with $1 \leq e_{i}<\ell$, let

$$
N^{\mathbf{e}}:=P_{1}^{e_{1}} P_{2}^{e_{2}} \cdots P_{t}^{e_{t}}
$$

Then any field in $\mathbf{A}_{t ; n}$ is of the form

$$
F_{N, \mathbf{e}}:=\mathrm{k}\left(\sqrt[\ell]{(-1)^{\operatorname{deg} N^{\mathbf{e}} N^{\mathbf{e}}}}\right) .
$$

Thus $\left|\mathbf{A}_{t ; n}\right|=(\ell-1)^{t-1} p(n, t)$. Note that if $F_{N, \mathbf{e}} \in \mathbf{A}_{t, e ; n}$ for some $\mathbf{e}$, then so in $F_{N, \mathbf{e}^{\prime}}$ for any $\mathbf{e}^{\prime}$. 
From now on we assume that $\ell \|(q-1)$. The general case is very hard to compute the rank of $M_{F}$ because of ambiguous ideal class without containing ambiguous ideal. Consider first the case that $\ell$ is odd, so that $a=1$ (See $\S 2$ ). It is shown in [13] that $M_{F}=\left(m_{i j}\right)$ is given by: $m_{i j}=\left(\frac{P_{i}}{P_{j}}\right)_{\ell}$, for $i \neq j$, where $(-)_{\ell}$ is the $\ell$-th power residue, and $m_{j j}$ is defined by the relation $\sum_{i} e_{i} m_{i j}=0$. Then, since $\frac{q-1}{\ell}$ is even or characteristic is 2 , from the $\ell$-th power reciprocity ([12, Theorem 3.3]), $M_{F}$ is symmetric. There is an algorithm to determine the number of $s \times s$ symmetric matrices with rank $r$ over $\mathbb{F}_{\ell}$ from the following proposition.

Proposition 4.1. Let $M$ be a symmetric $u \times u$ matrix of rank $r$ over $\mathbb{F}_{\ell}$. Let

$$
M_{1}=\left(\begin{array}{cc}
M & V \\
V^{T} & v
\end{array}\right),
$$

with $V \in \mathbb{F}_{\ell}^{u}, v \in \mathbb{F}_{\ell}$. Then among all possible $M_{1}$,

(i) $\ell^{r}$ of them have rank $r$.

(ii) $\ell^{r}(\ell-1)$ of them have rank $r+1$.

(iii) $\ell^{u+1}-\ell^{r+1}$ of them have rank $r+2$.

Suppose that we have given a total order ' $<$ ' on the set of monic irreducible polynomials in $\mathbb{A}$. For $N, N^{\prime} \in P(n, t)$, we say that $N$ and $N^{\prime}$ are equivalent if $\left(\frac{P_{j}}{P_{i}}\right)_{\ell}=\left(\frac{P_{j}^{\prime}}{P_{i}^{\prime}}\right)_{\ell}$, where $N=P_{1} \cdots P_{t}, P_{1}<\cdots<P_{t}$ and $N^{\prime}=P_{1}^{\prime} \cdots P_{t}^{\prime}$, $P_{1}^{\prime}<\cdots<P_{t}^{\prime}$. Let $\mathcal{N}(N)$ be the set of polynomials in $P(n, t)$, which are equivalent to $N$. Then it can be shown that (similar to $\S 3.1$ )

$$
|\mathcal{N}(N)| \sim \ell^{-\frac{t^{2}-t}{2}} \frac{q^{n}(\log n)^{t-1}}{(t-1) ! n} \sim \ell^{-\frac{t^{2}-t}{2}} p(n, t) .
$$

Note that we have $\ell^{-\frac{t^{2}-t}{2}}$ instead of $\ell^{-\frac{t^{2}+t}{2}}$ because we don't have any condition on $\operatorname{deg} P_{i}$, different from the classical case (formula (2.12) of [8]), where the condition $p_{i} \equiv p_{i}^{\prime} \bmod 4$ is imposed.

Let $\tilde{N}(t-1, u)$ be the number of $(t-1) \times(t-1)$ symmetric matrices with rank $u$. Then one can show as in $\S 2$ of [8] that

$$
\left|\mathbf{A}_{t, e ; n}\right|=(\ell-1)^{t-1} \tilde{N}(t-1, t-1-e)|\mathcal{N}(N)| .
$$

Then

$$
d_{t, e ; n}:=\frac{\left|\mathbf{A}_{t, e ; n}\right|}{\left|\mathbf{A}_{t ; n}\right|} \sim \ell^{-\frac{t^{2}-t}{2}} \tilde{N}(t-1, t-1-e),
$$

which is just the density $g(t, e)$ of $(t-1) \times(t-1)$ symmetric matrices with rank $t-1-e$ in the set of $(t-1) \times(t-1)$ symmetric matrices. We will compute the $\operatorname{limit}_{t \rightarrow \infty} g(t, e)$. From Proposition 4.1, we see that

$$
g(t+1, e)=\frac{1}{\ell^{e}} g(t, e-1)+\frac{\ell-1}{\ell^{1+e}} g(t, e)+\frac{\ell^{1+e}-1}{\ell^{1+e}} g(t, e+1), \text { if } e>0
$$


and

$$
g(t+1,0)=\frac{\ell-1}{\ell} g(t, 0)+\frac{\ell-1}{\ell} g(t, 1) .
$$

Let $G(t)=(g(t, 0), g(t, 1), \ldots, g(t, i), \ldots)$. One can show by induction that $G(t)$ converges to, as $t \rightarrow \infty$,

$$
G=\alpha\left(1, \frac{1}{\ell-1}, \ldots, \frac{1}{\prod_{i=1}^{k}\left(\ell^{i}-1\right)}, \ldots\right),
$$

where

$$
\alpha^{-1}=1+\frac{1}{\ell-1}+\frac{1}{(\ell-1)\left(\ell^{2}-1\right)}+\cdots .
$$

Now assume that $q \equiv 3 \bmod 4$ and $\ell=2$. Now the rest is almost the same as the classical case replacing ' $p \equiv 1 \bmod 4$ ' $(\operatorname{resp} . \quad p \equiv 3 \bmod 4)$ by ' $\operatorname{deg} P$ is even' (resp. odd). The reason for this is that the quadratic reciprocity for function field in this case is $\left(\frac{P_{i}}{P_{j}}\right)\left(\frac{P_{j}}{P_{i}}\right)=(-1)^{\operatorname{deg} P_{i} \operatorname{deg} P_{j}}$ in contrast with $\left(\frac{p_{i}}{p_{j}}\right)\left(\frac{p_{j}}{p_{i}}\right)=(-1)^{\frac{p_{i}-1}{2}} \frac{p_{j}-1}{2}$ in the classical case. Then following the same ideas to get Proposition 2.1 and Proposition 5.1 of [8], we get

$$
\begin{aligned}
& \left|\mathbf{A}_{t, e ; n}\right| \sim \sum_{\substack{1 \leq d \leq t \\
d \text { odd }}} N(t-1, d-1, t-1-e)\left(\begin{array}{l}
t \\
d
\end{array}\right) 2^{-\frac{t^{2}+t}{2}} \frac{q^{n}(\log n)^{t-1}}{(t-1) ! n} \text { for } n \text { odd, } \\
& \left|\mathbf{A}_{t, e ; n}\right| \sim \sum_{\substack{1 \leq d \leq t \\
d \text { even }}} N(t-1, d-1, t-1-e)\left(\begin{array}{l}
t \\
d
\end{array}\right) 2^{-\frac{t^{2}+t}{2}} \frac{q^{n}(\log n)^{t-1}}{(t-1) ! n} \text { for } n \text { even, }
\end{aligned}
$$

and

$$
\begin{aligned}
& d_{t, e ; n} \sim \sum_{\substack{1 \leq d \leq t \\
d \text { odd }}} N(t-1, d-1, t-1-e)\left(\begin{array}{l}
t \\
d
\end{array}\right) 2^{-\frac{t^{2}+t}{2}} \quad \text { for } n \text { odd } \\
& d_{t, e ; n} \sim \sum_{\substack{1 \leq d \leq t \\
d \text { even }}} N^{\prime}(t-1, d-1, t-1-e)\left(\begin{array}{l}
t \\
d
\end{array}\right) 2^{-\frac{t^{2}+t}{2}} \quad \text { for } n \text { even, }
\end{aligned}
$$

where $N(s, d, r)$ is the number of $s \times s$ matrices $M=\left(m_{i j}\right)$ over $\mathbb{F}_{2}$ with $m_{i j} \neq m_{j i}$ for $1 \leq i<j \leq d$ and with $m_{i j}=m_{j i}$ for $d+1 \leq i \leq s$ and $1 \leq j \leq s$ such that $\operatorname{rank}(M)=r$, and $N^{\prime}(s, d, r)$ is the number of $s \times(s+1)$ matrices $\overline{M^{\prime}}$ whose first column is the transpose of the vector $(1, \ldots, 1,0, \ldots, 0)$ with first $d$ entries 1 and the rest part is an $s \times s$ matrix $M^{\prime}=\left(m_{i j}^{\prime}\right)$ over $\mathbb{F}_{2}$ with $m_{i j}^{\prime} \neq m_{j i}^{\prime}$ for $1 \leq i<j \leq d$ and $m_{i j}^{\prime}=m_{j i}^{\prime}$ for $d+1 \leq i \leq s$ and $1 \leq j \leq s$ such that $\operatorname{rank}\left(\overline{M^{\prime}}\right)=r$. Then as in $[8, \S 4, \S 5]$,

$$
G(t):=\left(d_{t, 0,2 n+1}, d_{t, 1,2 n+1}, \ldots\right) \quad \text { and } \quad G^{\prime}(t):=\left(d_{t, 0,2 n}^{\prime}, d_{t, 1,2 n}^{\prime}, \ldots\right)
$$

converge to $\frac{Y}{2}$ and $\frac{Y^{\prime}}{2}$ as $t \rightarrow \infty$, where

$$
Y=\left[\prod_{m=1}^{\infty}\left(1-2^{-m}\right)\right]^{-1}\left(1,2, \ldots, 2^{-i^{2}} \prod_{m=1}^{i}\left(1-2^{-m}\right)^{-2}, \ldots\right)
$$


and

$Y^{\prime}=\left[\prod_{m=2}^{\infty}\left(1-2^{-m}\right)\right]\left(1,2 / 3, \ldots, 2^{-i(i+1)} \prod_{m=1}^{i}\left(1-2^{-m}\right)^{-1}\left(1-2^{-m-1}\right)^{-1}, \ldots\right)$.

\section{Generalization to $\ell^{m}$-cyclic function fields}

In this section we consider $\ell^{m}$-cyclic extensions $F$ of $\mathrm{k}$ and the following question as in [9]: how likely is $\lambda_{2}^{+}(F)=0, \lambda_{2}^{+}(F)=1, \lambda_{2}^{+}(F)=2, \ldots$ ? When $m=1$, its answer is already obtained in $\S 4$. So we assume $m \geq 2$. Assume that we are given integers $m_{1}, \ldots, m_{t}$ such that $m=m_{1} \geq m_{2} \geq \cdots \geq m_{t} \geq 1$. Let $\Delta$ be the abelian group of type $\left(\ell^{m_{2}}, \ldots, \ell^{m_{t}}\right)$ (When $t=1$, we let $\Delta$ be the trivial group).

Write $\mathbf{A}(\Delta)$ for the set of all $F$ as above such that the narrow genus group $\mathrm{Cl}^{+}(F)_{\ell} / \mathrm{Cl}^{+}(F)_{\ell}^{1-\sigma}$ is isomorphic to $\Delta$, and

$$
\begin{aligned}
& \mathbf{A}(\Delta)_{n}:=\{F \in \mathbf{A}(\Delta): \operatorname{deg}(\operatorname{cond}(F))=n\}, \\
& \mathbf{A}_{e}(\Delta):=\left\{F \in \mathbf{A}(\Delta): \lambda_{2}^{+}(F)=e\right\}, \\
& \mathbf{A}_{e}(\Delta)_{n}:=\mathbf{A}_{e}(\Delta) \cap \mathbf{A}(\Delta)_{n} .
\end{aligned}
$$

Then we define the density $d_{e}(\Delta)$ of $\mathbf{A}_{e}(\Delta)$ in $\mathbf{A}(\Delta)$ by

$$
d_{e}(\Delta):=\lim _{n \rightarrow \infty} \frac{\left|\mathbf{A}_{e}(\Delta)_{r n}\right|}{\left|\mathbf{A}(\Delta)_{r n}\right|} .
$$

It is easy to see that for any ordering $\left(m_{j_{i}}\right)$ of $m_{1}, \ldots, m_{t}$ and monic irreducible polynomials $P_{1}, \ldots, P_{t}$ with $q^{\operatorname{deg} P_{i}} \equiv 1 \bmod \ell^{m_{j_{i}}}$, there are

$$
\frac{\prod_{i=1}^{t}\left(\ell^{m_{j_{i}}}-\ell^{m_{j_{i}}-1}\right)}{\left(\ell^{m}-\ell^{m-1}\right)}=\prod_{i=2}^{t}\left(\ell^{m_{i}}-\ell^{m_{i}-1}\right)
$$

distinct fields $F$ in $\mathbf{A}(\Delta)$ such that the conductor of $F$ is $P_{1} \cdots P_{t}$ and each $P_{i}$ has ramification index $\ell^{m_{j_{i}}}$ in $F$. So we have

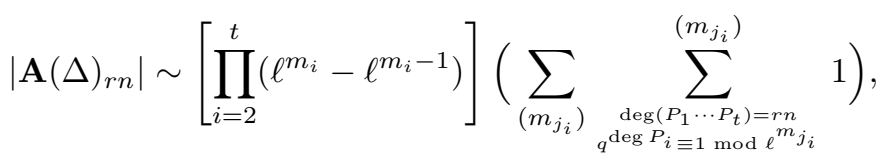

where $\sum_{\left(m_{j_{i}}\right)}$ denotes a sum over all distinguishable orderings of $m_{1}, \ldots, m_{t}$, and $\sum^{\left(m_{j_{i}}\right)}$ is a sum for a fixed reordering $\left(m_{j_{i}}\right)$. For any positive integer $k$, write $r_{k}$ for the smallest positive integer such that $\ell^{k} \mid q^{r_{k}}-1$. Then for any monic irreducible polynomial $P$ in $\mathbb{A}$, we have $q^{\operatorname{deg} P} \equiv 1 \bmod \ell^{k}$ if and only if $r_{k} \mid \operatorname{deg} P$. Following the method of $[11, \S 9]$, we have

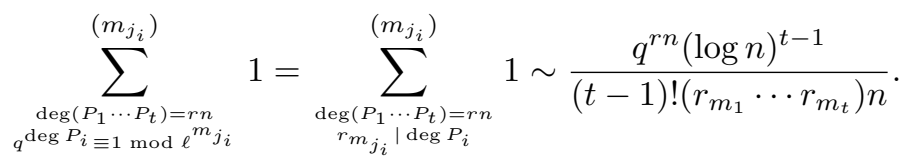


Let $v_{w}=\left|\left\{m_{i}: m_{i}=w\right\}\right|$ for $1 \leq w \leq m$. Since there are $\frac{t !}{\left(v_{1} !\right) \cdots\left(v_{m} !\right)}$ distinguishable orderings $\left(m_{j_{i}}\right)$ of $m_{1}, \ldots, m_{t}$, by (5.5) and (5.6), we have

$$
\left|\mathbf{A}(\Delta)_{r n}\right| \sim \frac{t \prod_{i=2}^{t}\left(\ell^{m_{i}}-\ell^{m_{i}-1}\right)}{\left(r_{m_{1}} \cdots r_{m_{t}}\right)\left(v_{1} !\right) \cdots\left(v_{m} !\right)} \frac{q^{r n}(\log n)^{t-1}}{n} .
$$

Now we are going to obtain an asymptotic formula for $\mathbf{A}_{e}(\Delta)_{r n}$. Assume first that $r>1$, that is $\ell \nmid q-1$. Following the arguments in [3, $\S 5$, Theorem 5.3], one can associate a $t \times(t-1)$ matrix $\bar{M}_{F}^{\prime}$ to $F$ such that $\lambda_{2}^{+}(F)=t-1-\operatorname{rank}\left(\bar{M}_{F}^{\prime}\right)$. Moreover, as in $[9, \S 2]$, one can replace the matrix $\bar{M}_{F}^{\prime}$ with a $t \times t$ matrix $\bar{M}_{F}$ such that $\operatorname{rank}\left(\bar{M}_{F}^{\prime}\right)=\operatorname{rank}\left(\bar{M}_{F}\right)$. Especially, if $F \in \mathbf{A}_{e}(\Delta)$, then the matrix $\bar{M}_{F}$ has rank $t-1-e$. Then $\left|A_{e}(\Delta)_{r n}\right|$ can be estimated as

$$
\left|\mathbf{A}_{e}(\Delta)_{r n}\right| \sim \sum_{\Gamma} \sum_{\operatorname{rank}(\Gamma)=t-1-e} \sum_{\substack{\left(m_{j_{i}}\right) \\ \operatorname{deg}\left(P_{1} \ldots P_{t}\right)=r n \\ r m_{j_{i}} \mid \operatorname{deg} P_{i}}}^{\left(m_{j_{i}}\right)} \sum_{\substack{F \\ \operatorname{cond}(F)=P_{1} \cdots P_{t}}} \delta_{\Gamma},
$$

where the first sum is over all $t \times t$ matrices $\Gamma$ over $\mathbb{F}_{\ell}$ with $\operatorname{rank} t-1-e$. The fourth sum runs over all $F \in \mathbf{A}(\Delta)$ with conductor $P_{1} \cdots P_{t}$ such that each $P_{i}$ has ramification index $\ell^{m_{j_{i}}}$, and $\delta_{\Gamma}=1$ if $\bar{M}_{F}=\Gamma$ and $\delta_{\Gamma}=0$ otherwise. If the ordering $\left(m_{j_{i}}\right)$ has $m_{j_{i}}=m_{i}$ for $1 \leq i \leq t$, then $\bar{M}_{F}$ has the following form:

$$
M_{F}=\left(\begin{array}{cc}
M_{1} & M_{2} \\
O & D
\end{array}\right)
$$

where $M_{1}$ is a $v_{m} \times v_{m}$ matrix over $\mathbb{F}_{\ell}$ in which the sum of entries in each row is zero, $M_{2}$ is a $v_{m} \times\left(t-v_{m}\right)$ matrix over $\mathbb{F}_{\ell}, O$ is the $\left(t-v_{m}\right) \times v_{m}$ zero matrix and $D$ is a $\left(t-v_{m}\right) \times\left(t-v_{m}\right)$ diagonal matrix.

Let $\Gamma$ be a $t \times t$ matrix over $\mathbb{F}_{\ell}$ such that $\Gamma$ has the same form as the matrix on the right hand side of (5.9), and let

$$
N(\Gamma)=\sum_{\substack{\operatorname{deg}\left(P_{1} \cdots P_{t}\right)=r n \\ r_{i} \mid \operatorname{deg} P_{i}}} \sum_{\substack{F \\ \operatorname{cond}(F)=P_{1} \cdots P_{t}}} \delta_{\Gamma},
$$

where $\delta_{\Gamma}=1$ if $\bar{M}_{F}=\Gamma$ and $\delta_{\Gamma}=0$ otherwise. Following the idea of $[9, \S 2]$ and adopting the similar method as in $\S 3.1$, we get:

Proposition 5.1. We have

$$
N(\Gamma) \sim \frac{\prod_{i=2}^{t}\left(\ell^{m_{i}}-\ell^{m_{i}-1}\right)}{\left(r_{m_{1}} \cdots r_{m_{t}}\right) \ell^{v_{m}(t-1)+t-v_{m}}} \frac{q^{r n}(\log n)^{t-1}}{(t-1) ! n},
$$

and so

$$
\begin{aligned}
\left|\mathbf{A}_{e}(\Delta)_{r n}\right| \sim & \frac{t N\left(t, v_{m}, t-1-e\right)\left(\ell^{m}-\ell^{m-1}\right)^{v_{m}-1} \prod_{i=v_{m}+1}^{t}\left(\ell^{m_{i}}-\ell^{m_{i}-1}\right)}{\left(r_{m_{1}} \cdots r_{m_{t}}\right)\left(v_{1} !\right) \cdots\left(v_{m} !\right) \ell^{v_{m}(t-1)+t-v_{m}}} \\
& \times \frac{q^{r n}(\log n)^{t-1}}{n},
\end{aligned}
$$


where $N\left(t, v_{m}, t-1-e\right)$ denote the number of $\Gamma^{\prime}$ 's as above with $\operatorname{rank}(\Gamma)=$ $t-1-e$.

Finally, by (5.7) and (5.10), we have

$$
d_{e}(\Delta)=\frac{N\left(t, v_{m}, t-1-e\right)}{\ell^{v_{m}(t-1)+t-v_{m}}} \quad \text { for } 0 \leq e \leq t-1 .
$$

We note that the number $N\left(t, v_{m}, t-1-e\right)$ can be computed as in Lemma 2.4 and the remark following it in [9].

Now suppose that $r=1$. As before we only consider the case $\ell \|(q-1)$. Let $\mathbf{B}_{t}$ be the set of all $\ell^{m}$-cyclic extensions $F$ of $\mathrm{k}$ such that $t$ finite primes ramify in $F / \mathrm{k}$, and

$$
\begin{aligned}
& \mathbf{B}_{t ; n}:=\left\{F \in \mathbf{B}_{t}: \operatorname{deg}(\operatorname{cond}(F))=n\right\} \\
& \mathbf{B}_{t, e}:=\left\{F \in \mathbf{B}_{t}: \lambda_{2}^{+}(F)=e\right\} \\
& \mathbf{B}_{t, e ; n}:=\mathbf{B}_{t, e} \cap \mathbf{B}_{t ; n} .
\end{aligned}
$$

Then as in [9] we see that the density $d_{t, e}:=\lim _{n \rightarrow \infty} \frac{\left|\mathbf{B}_{t, e ; r n}\right|}{\left|\mathbf{B}_{t ; r n}\right|}$ is given by

$$
d_{t, e}=\frac{\sum_{u=1}^{t} \frac{N(t, u, t-1-e)}{\ell^{u(t-1)+t-u}}\left(\begin{array}{l}
t \\
u
\end{array}\right) \frac{(m-1)^{t-u}}{m^{t}}}{1-\left(\frac{m-1}{m}\right)^{t}},
$$

and its limit $d_{\infty, e}:=\lim _{t \rightarrow \infty} d_{t, e}=0$.

The formula (5.7) also works in this case too. As in the case of $r>1$, we get the matrix $M_{F}$ in (5.9). But in this case we need one more step, that is, multiply $z_{i}$, which is defined as in (2.12) of [9], to each $i$ th row of $M_{F}$ with $1 \leq i \leq v_{m}$. The resulting matrix $M_{F}^{*}$ has the same rank as $M_{F}$ and is of the form

$$
M_{F}^{*}=\left(\begin{array}{cc}
M_{1}^{*} & M_{2}^{*} \\
O & D
\end{array}\right)
$$

where $M_{1}^{*}$ is a $v_{m} \times v_{m}$ symmetric matrix over $\mathbb{F}_{\ell}$ in which the sum of entries in a row is 0 and $M_{2}^{*}, O, D$ are the same as before. When $\ell=2$ as in the classical case, we do not need this step, since $z_{i}=1$ in this case. The reason for the symmetricity of $M_{1}^{*}$ follows from the $\ell$ th power reciprocity law ([12], Theorem 3.3) for $\ell>2$, and from the fact that $m>1$ for $\ell=2$. Exactly the same way as in the case $r>1$, we obtain

$$
\left|\mathbf{A}_{e}(\Delta)_{n}\right| \sim \frac{N_{s}\left(t, v_{m}, t-1-e\right) \prod_{i=2}^{t}\left(\ell^{m_{i}}-\ell^{m_{i}-1}\right)}{\left(r_{m_{1}} \cdots r_{m_{t}}\right) v_{1} ! \cdots v_{m} ! \ell^{\frac{v_{m}\left(v_{m}-1\right)}{2}}+v_{m}\left(t-v_{m}\right)} \frac{t q^{n}(\log n)^{t-1}}{n}
$$

where $N_{s}(t, u, s)$ denotes the number of matrices $\Gamma$ of the form specified in (5.16) such that $\operatorname{rank} \Gamma=s$. The density $d_{e}(\Delta)$ and $d_{t, e}$ are given by

$$
d_{e}(\Delta)=\frac{N_{s}\left(t, v_{m}, t-1-e\right)}{\ell^{\frac{v_{m}\left(v_{m}-1\right)}{2}+v_{m}\left(t-v_{m}\right)}},
$$


and

$$
d_{t, e}=\left[\sum_{u=1}^{t} \frac{N_{s}(t, u, t-1-e)}{\ell^{\frac{u(u-1)}{2}}+u(t-u)}\left(\begin{array}{l}
t \\
u
\end{array}\right) \frac{(m-1)^{t-u}}{m^{t}}\right]\left(1-\left(\frac{m-1}{m}\right)^{t}\right)^{-1}
$$

\section{References}

[1] B. Angles, On Hilbert class field towers of global function fields, Drinfeld modules, modular schemes and applications (Alden-Biesen, 1996), 261-271, World Sci. Publ., River Edge, NJ, 1997.

[2] S. Bae and J. Koo, Genus theory for function fields, J. Austral. Math. Soc. Ser. A 60 (1996), no. 3, 301-310.

[3] A. Fröhlich, Central Extensions, Galois Groups, and Ideal Class Groups of Number Fields, American Mathematical Society, Providence, RI, 1983.

[4] F. Gerth, Number fields with prescribed $\ell$-class groups, Proc. Amer. Math. Soc. 49 (1975), 284-288.

[5] _ Asymptotic behavior of number fields with prescribed $\ell$-class numbers, J. Number Theory 17 (1983), no. 2, 191-203.

[6] _ Counting certain number fields with prescribed $\ell$-class numbers, J. Reine Angew. Math. 337 (1982), 195-207.

[7] _ An application of matrices over finite fields to algebraic number theory, Math. Comp. 41 (1983), no. 163, 229-234.

[8] _ The 4-class ranks of quadratic fields, Invent. Math. 77 (1984), no. 3, 489-515.

[9] Densities for certain $\ell$-ranks in cyclic fields of degree $\ell^{n}$, Compositio Math. 60 (1986), no. 3, 295-322.

[10] Densities for ranks of certain parts of p-class groups, Proc. Amer. Math. Soc. 99 (1987), no. 1, 1-8.

[11] J. Knopfmacher, Analytic Arithmetic of Algebraic Function Fields, Marcel Decker Inc., New York-Basel, 1979.

[12] M. Rosen, Number Theory in Function Fields, GTM vol. 210, Springer, 2002.

[13] C. Wittmann, $\ell$-Class groups of cyclic function fields of degree $\ell$, Finite Fields Appl. 13 (2007), no. 2, 327-347.

Sunghan Bae

Department of Mathematics

Korea Advanced Institute of Science and Technology

DAEJEON 305-701, Korea

E-mail address: shbae@kaist.ac.kr

Hwanyup Jung

Department of Mathematics Education

Chungbuk National University

Cheonguu 361-763, Korea

E-mail address: hyjung@chungbuk.ac.kr 